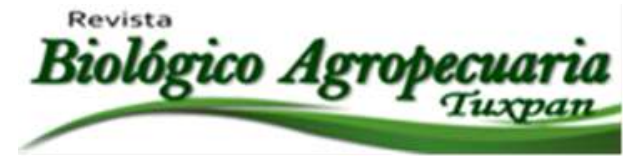

\title{
Análisis descriptivo de la industria de muebles de madera de Delicias, Chihuahua
}

Descriptive analysis of the wood furniture industry of Delicias, Chihuahua

\author{
Magaña Magaña José Eduardo ${ }^{\bowtie 1}$, Talamantes González Thalia Fernanda ${ }^{1}$, Villarreal Ramírez Víctor \\ Hugo $^{1}$, Kiessling Davison Christian Mauricio ${ }^{1}$, Palacios Monarrez Abdoón ${ }^{1}$ \\ ${ }^{1}$ Facultad de Ciencias Agrícolas y Forestales de la Universidad Autónoma de Chihuahua \\ ${ }^{凶}$ Autor para correspondencia: emagana@uach.mx
}

Recibido: $18 / 04 / 2020$

Aceptado: 24/05/2020

\section{RESUMEN}

La industria de fabricantes de muebles de madera contribuye de manera importante a la generación de empleos de la región de Delicias, Chihuahua. La muestra con la que se logró la presente investigación fueron empresas representativas de la industria como lo demuestra el hecho de que el promedio de antigüedad en el mercado fue de 30 años. Se determinó las capacidades y necesidades productivas. Se aplicó un cuestionario de 35 preguntas con 95 variables, aplicados a 25 empresas. De los cuales el $40 \%$ son microempresas y el 53\% son pequeñas empresas. El total de profesionistas en la muestra fue de 8.24\% del número total de trabajadores que laboran principalmente en el área administrativa. El 93\% de las empresas no cuentan con un plan estratégico lo que representó una gran debilidad. Entre las principales fortalezas fueron que el 53\% cuenta con una antigüedad en el mercado de 31 a 40 años, lo que significó que tienen experiencia en la industria y cuentan con capacidad emprendedora y potencial para transformar de manera competitiva en diseño y calidad sus productos. Lo anterior manifestó que se tiene que trabajar más en capacitación en las áreas de producción, calidad y manejo de desechos, en planeación estratégica, en la norma ISO 035, en uso de suelo e integración a las diferentes asociaciones empresariales.

Palabras clave: Habilidades, recursos, planeación, capacidades, emprendedores.

\begin{abstract}
The wood furniture industry is very important because it has been contributing in generating jobs in Delicias, Chihuahua. The sample used in this research, represents all the business of this industry. The information gave us an average of 30 years in the market place. It was determined the capacities and productive needs. A questionnaire with 95 variables was applied to 25 different wood furniture companies. $40 \%$ of them were micro-enterprises and $53 \%$ smaller. The total of professionals were $8.24 \%$ of the total number of workers. They mainly worked in the administrative area. $93 \%$ of companies do not have a strategic plan, which represented a great weakness for the companies in this industry. The main strengths found were that the 53\% of them had been in the market between 31 to 40 years, it means that they have had a lot of experience in the industry and having the entrepreneurial capacity and potential to competitiveness to transform their products into design and most definitely quality. Like previously mentioned, more work has to be done in training in the production areas, quality and waste management. Also in strategic planning, in the ISO 035 standard and finally in the land use and the integration into different business associations.
\end{abstract}


Keywords: ability, resources, capacities, entrepreneurship, strategic planning.

\section{INTRODUCCIÓN}

Existen factores económicos, de mercado y financieros que limitan la integración y la fortaleza para el crecimiento y desarrollo de la industria mueblera ya que tiene deficiencias estructurales que no ha podido superar en las últimas décadas. La caracterización del sector no es competitiva, es extremadamente ineficiente, el mercado interno es muy débil y la competencia de productos baratos del exterior aumenta cada día (Espinosa Gómez 2012). Por lo tanto, es necesario identificar las principales necesidades de la industria del mueble. Por este motivo, es necesario realizar un análisis estratégico que se integre en la cadena de valor agregado e identifique estos factores y luego busque soluciones básicas e innovadoras que nos permitan desarrollar un entorno más motivador. La calidad de la producción de muebles es tal que proporciona solidez en el crecimiento y desarrollo de la región de Delicias Chihuahua. Es un mercado enorme con grandes oportunidades para importadores y exportadores, fabricantes, diseñadores y distribuidores.

La industria del mueble ha sufrido cambios en su desarrollo, muchos de los cuales han sido motivados por la falta de estructura en las pequeñas empresas ya que esta es una de las actividades económicas más importantes de la ciudad. En las últimas décadas, se han experimentado mayores tasas de crecimiento de la industria, así como un crecimiento constante en el sector manufacturero. Aparentemente, el desarrollo de esta industria está generando cambios incontrolados en el uso del suelo urbano ciudad. La industria mueblera de
México se considera no solo como una industria sino como una tradición familiar, aunque considerada relativamente joven, con poca resistencia al cambio y un estilo predominantemente artesanal. El nivel de calidad del mueble mexicano, hasta hace algunos años era el adecuado para el mercado nacional; sin embargo, para mantenerse en éste y estar en posibilidades de competir con el mercado internacional, el sector ha requerido aceptar sus procesos y su mentalidad (Secofi, 1998) citado por González et al (2016).

Por lo tanto, es importante saber cómo o de qué manera la expansión o crecimiento de la industria del mobiliario ha influido en la estructura urbana de la ciudad. La planeación estratégica es un proceso participativo estructurado y manejado sistemáticamente. A través de este proceso, grupos de interés e individuos activos (organizaciones, comunidades, entre otros) buscan entre los posibles ambientes resultados deseables para ellos mismos y al mismo tiempo la estrategia para alcanzar dichos resultados (Magaña et al 2003).

Así mismo, Bracker y Pearson (1986) citado por López (2015); definen que las PyMES, pequeñas y medianas empresas con mayor éxito son las que están más conscientes de que existan herramientas de planeación estratégica en las empresas y al planear sus actividades. En gran mayoría los empresarios de las Pymes han adopta algún tipo de estrategia, ya sea para adaptarse al medio ambiente interno o externo que esté viviendo en la actualidad o para la prevención de acontecimientos futuros; es un porcentaje mínimo de empresas con "estrategias" bien establecidas en su proceso 
productivo (Velarde et al 2011). En este sentido, la planeación estratégica juega un papel fundamental en las empresas, de modo que se fundamente en los objetivos generales y específicos en marco de los planes integrales de desarrollo fijados en un tiempo determinado, que no sean corto para así no quedar con deudas en el camino, ni demasiado largo que contemple la posibilidad de perderse (Daza L. Javier 2010).

Ya sea que se les denomine con el nombre de planeación estratégica o no, las pequeñas empresas diseñan su propia estructura para así llevar un control de sus recursos tanto económicos, humanos y de materia prima, no obstante esta falta de diseño bien estructurado es la responsable de perdidas incuantificables que llevan al declive de estas MIPyMES. La industria Mueblera de nuestra región no cuenta con cifras actualizadas que dimensionen la posición y mercado de competencia, por lo tanto, no cuentan con datos que nos permitan generar estrategias y un plan de acción que permita obtener resultados confiables para ver el impacto en el PIB estatal y nacional para un desarrollo confiable en esta industria.

Es necesario obtener información actualizada, útil y disponible de este sector, para medir sus capacidades y necesidades. Para diseñar y aplicar un plan de acción que permita obtener resultados confiables que nos muestre el impacto en el PIB estatal y nacional, y poder conocer y cuantificar sus necesidades actuales. Con esta información, el sector podrá planificar y proyectar un desarrollo sostenible que se traduzca en mayores ingresos económicos y un crecimiento sostenido para el sector mueblero de la región centro-sur de Chihuahua, además de lanzar un nuevo ciclo en el que se sugiere que la información sea nueva y se mantenga una constante retroalimentación para reducir la incertidumbre en la toma de decisiones futuras.
Por lo tanto, el fin de la realización de la presente investigación es determinar y analizar las capacidades y necesidades que impacten en el desarrollo y desempeño de la industria de fabricación de muebles en la región de Delicias Chihuahua. Realizando una investigación donde se cuantifiquen las capacidades de las empresas manufactureras de, considerando: capital humano productivo y administrativo, capital de recursos productivos y activos no tangibles, capital financiero, contable, capital de comercialización y capital relacional. Y así mismo cuantificar las necesidades de las empresas tomando en cuenta: producción, capacitación, créditos, comercialización y manejo de residuos. Y así poder presentar los resultados del presente estudio a las partes interesadas de la industria manufacturera de muebles de Delicias Chihuahua.

\section{MATERIALES Y MÉTODOS}

La investigación se realizó en el Municipio de Delicias, Chihuahua. Con un método de muestreo basado en la utilización de censo, aplicado a todos los fabricantes de muebles para identificar las necesidades en la industria Mueblera. Con una población objeto de estudio de 15 fabricantes de muebles del Municipio de Delicias, Chihuahua. La metodología que se utilizo fue en base en encuesta, donde Se aplicó un cuestionario, las preguntas se diseñaron para cuantificar los factores que impiden que la industria de la fabricación de muebles en Delicias, Chihuahua se desarrolle.

El procesamiento de la información recabada por el encuestador se realizó análisis de frecuencias, clusters, chaid, tablas cruzadas. Como herramienta principal se utilizará el programa estadístico SPSS versión 23. Las fuentes de información fueron 2 las primarias: fabricantes de muebles de madera en la región 
de Delicias, Chihuahua y las secundarias: Se utilizó el mapeo territorial con el método de la bola de nieve de toda la ciudad por un encuestador. El tipo de investigación fue exploratoria y correlacional con 93 variables de las cuales 37 son cuantitativas y 56 cualitativas.

\section{RESULTADOS}

En la presente investigación se pretende generar un análisis empresarial de las capacidades y de las necesidades empresariales de las pequeñas y medianas industrias Muebleras del Municipio de Delicias para generar estrategias que mejoren el desarrollo y desempeño de la industria de fabricación de muebles en dicho Municipio.

Se aplicó un cuestionario a 25 empresas con 93 variables de las cuales 37 son cuantitativas y 56 cualitativas. El método de aplicación fue de entrevista personal, por teléfono y por correo. La técnica de muestreo utilizada fue la bola de nieve. Los principales resultados son los siguientes:

Lo primero que se realizó fue medir la confiabilidad de la escala de Likert de las preguntas del cuestionario aplicado para lo cual, se utilizó el criterio general de George y Mallery (2003) que sugieren las recomendaciones siguientes para evaluar los coeficientes de alfa de Cronbach:

Coeficiente alfa $>.9$ es excelente; Coeficiente alfa $>.8$ es bueno; Coeficiente alfa $>.7$ es aceptable; Coeficiente alfa

$>.6$ es cuestionable; Coeficiente alfa $>.5$ es pobre

En el caso de esta investigación, el Alpha es 0.763 y de acuerdo al criterio general de George Mallery es aceptable cerca a bueno en las escalas Likert que se utilizaron. Como se observa en el cuadro 1, en donde se calculó el coeficiente

Cronbach.

Cuadro 1. Análisis de fiabilidad del instrumento

\begin{tabular}{|l|l|}
\hline $\begin{array}{l}\text { Alfa de } \\
\text { Cronbach }\end{array}$ & $\begin{array}{l}\mathrm{N} \\
\text { elementos }\end{array}$ \\
\hline .768 & 42 \\
\hline
\end{tabular}

Fuente: Elaboración propia.

Las micro empresas, pequeñas y medianas empresas, las MIPyMES, son base fundamental para la economía de cualquier país son los que hacen competitivo a un país, generando el incremento a nuevas inversiones y fortalecen las industrias (Carriedo Carlos 2017). Julio A. Santaella, presidente del INEGI, señaló que en México 4.1 millones de empresas son micro, pequeñas o medianas y gran parte de la actividad económica y el empleo está estrechamente ligado a ellas, generando $72 \%$ del empleo y portan52\% del Producto Interno Bruto (INEGI 2019).

El 40\% de las empresas que participaron en el estudio se clasifican como Microempresas, el $53 \%$ como pequeñas empresas y el $7 \%$ fueron medianas empresas (Grafica 1). 
Grafica 1. Clasificacion de MIPYMES de acuerdo al numero de trabajadores
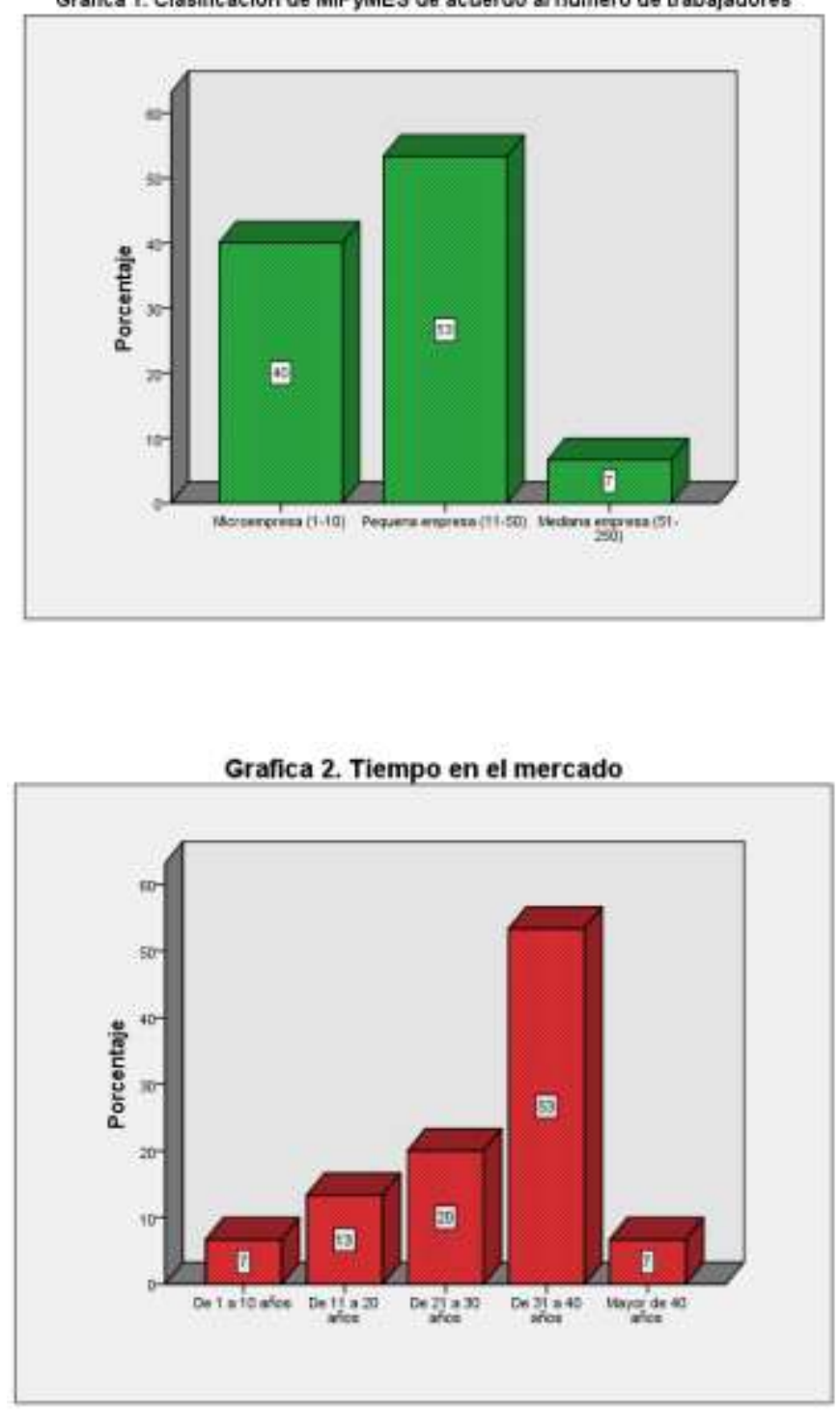

El 7\% de las empresas que fueron objeto de estudio permanecen en el mercado con 1 a 10 años de antigüedad, el $13 \%$ con 11 a 20 años de antigüedad, el $20 \%$ con 21 a 30 años de antigüedad, el 53\% con 31 a 40 años y el 7\% fueron mayores a 40 años de antigüedad (Gráfica 2). 


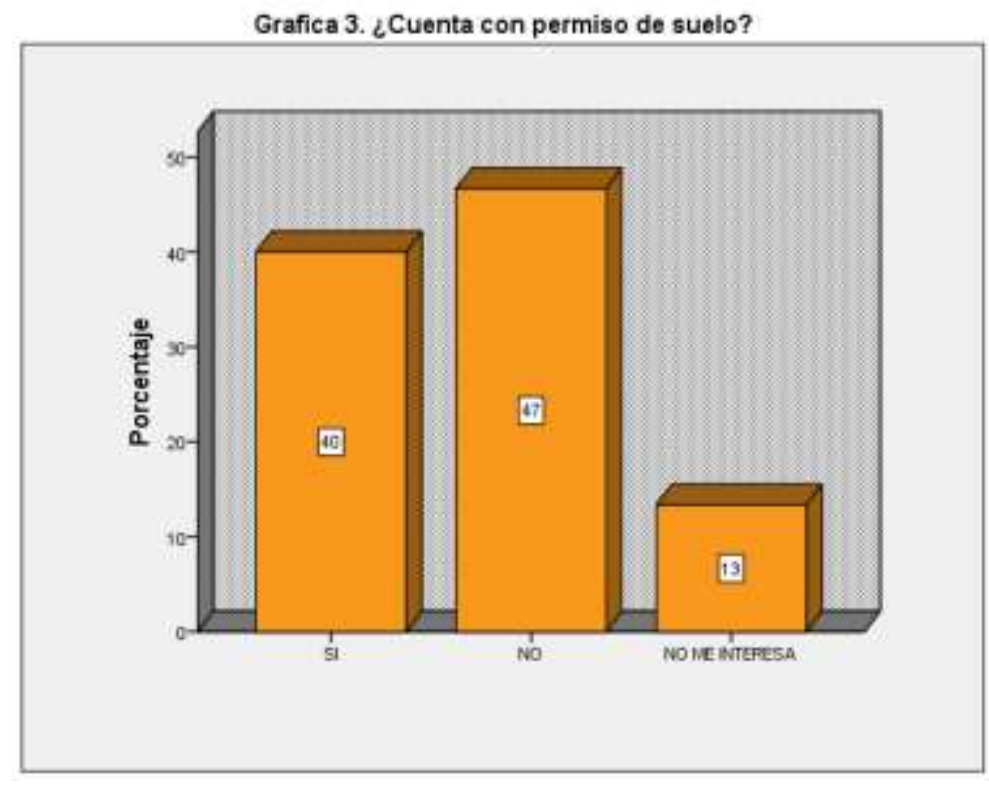

El $47 \%$ de los muebleros que participaron en el tienen un interés en contar con permiso de suelo estudio no cuentan con permiso de suelo, el (Gráfica 3). $40 \%$ cuentan con permiso de suelo y el $13 \%$ no

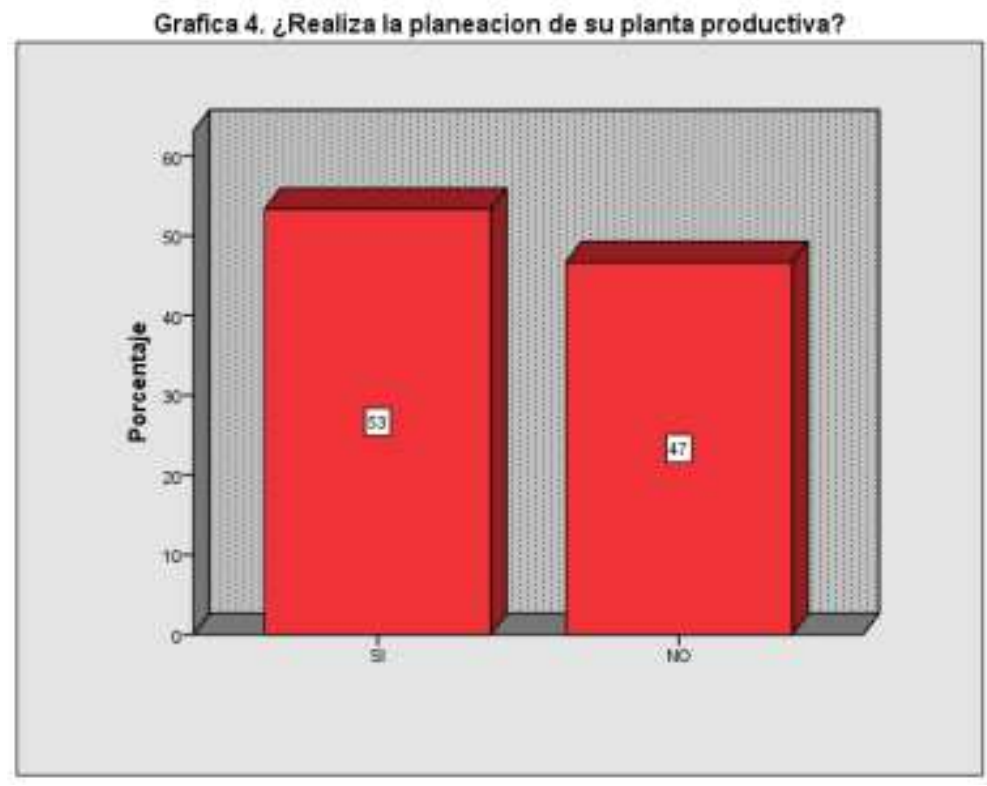

El 53\% de las empresas que participaron en el estudio si realizan planeación en su planta productiva y el $47 \%$ no cuentan con una planeación en su planta productiva (Gráfica 4). 
Grafica 5. ¿Esta usted capacitado para la norma 10S035?

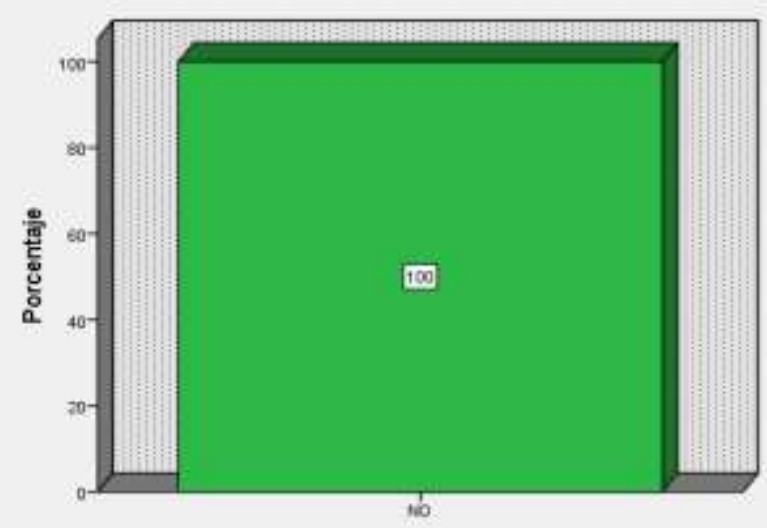

El $100 \%$ de las empresas que formaron parte en el estudio no cuentan con una capacitación en la norma ISO 035. Esto es un signo de que no tienen información del significado y de la importancia de aplicar esta norma vigente desde el 2019. Se manifiesta con mayor certidumbre que la falta de información es que solo el $13 \%$ de las empresas que participaron en el estudio, son parte de la asociación de fabricantes de muebles de Delicias y el $87 \%$ no forman parte de esa asociación (Gráficas 5 y 6).

Grafica 6. ¿Es usted miembro de la asociación de fabricantes de muebles?

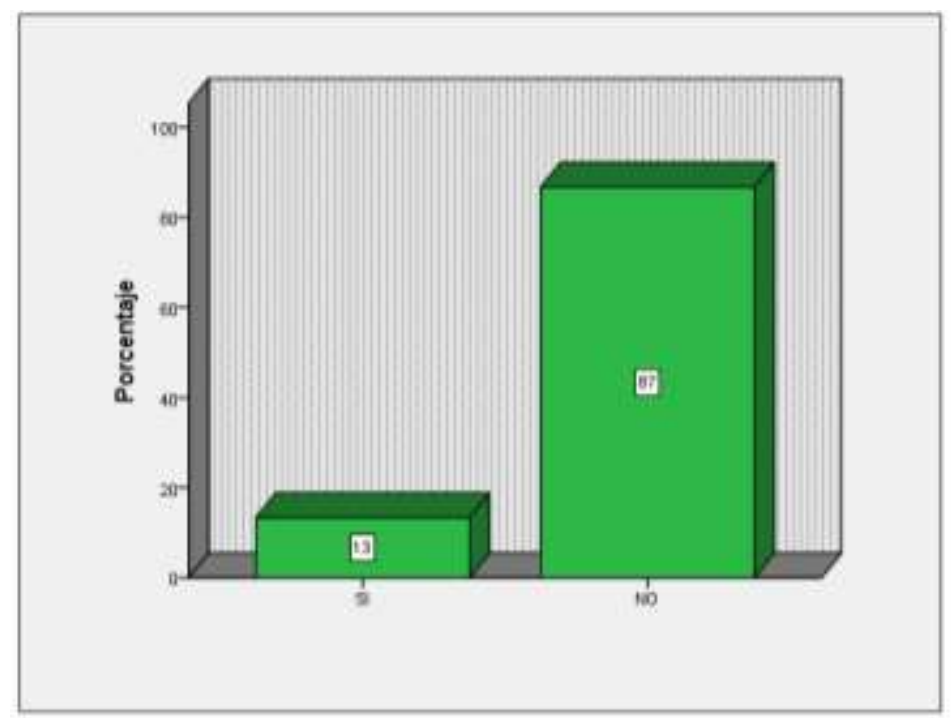

Cuadro 2. Total de empleados 


\begin{tabular}{|c|c|}
\hline $\begin{array}{lr}\text { N } & \text { Válidos } \\
\text { Media } & \text { Perdidos } \\
\text { Rango } & \\
\text { Mínimo } & \\
\text { Máximo } & \\
\text { Suma } & \end{array}$ & $\begin{array}{l}15 \\
0 \\
18.60 \\
60 \\
4 \\
64 \\
279\end{array}$ \\
\hline
\end{tabular}

Fuente: Elaboración propia

Cuadro 3. ¿Cuánto tiempo tiene en el negocio de la producción de muebles en años?

\begin{tabular}{|ll|l|}
\hline & \multicolumn{1}{|c|}{ Válidos } & 15 \\
Nedia & Perdidos & 0 \\
Rango & & 30.00 \\
Mínimo & 37 \\
Máximo & 5 \\
Suma & 42 \\
\hline
\end{tabular}

Fuente: Elaboración propia.

A pesar de que las empresas enfocadas en la muestra, tienen una antigüedad en el mercado promedio de 30 años en donde la más reciente manifestó 5 años y la más antigua 42 años, el promedio de empleados es de 18.60 por empresa (cuadro 2). Esto nos conduce a una conclusión que a pesar de la antigüedad y del conocimiento de mercado, no se observa una correlación entre la antigüedad y el número de empleados cómo se observa en el cuadro 4, en la prueba de Chi-cuadrada.

Cuadro 4. Pruebas de chi-cuadrado

\begin{tabular}{|l|l|l|l|}
\hline & Valor & gl & Sig. asintótica (bilateral) \\
\hline Chi-cuadrado de Pearson & $9.107^{\mathrm{a}}$ & 12 & .694 \\
Razón de verosimilitudes & 10.317 & 12 & .588 \\
Asociación lineal por lineal & .387 & 1 & .534 \\
N de casos válidos & 15 & & \\
\hline
\end{tabular}

Fuente: Elaboración propia. 
Ho: no existe correlación o son independientes las variables de tiempo en el mercado y empresas por número de empleados

Ha: si existe correlación y están asociados las variables de tiempo en el mercado y empresas por número de empleados

Chi-cuadrada calculada $=9.107, \mathrm{gl}=12$
Chi de tablas $=21.03 ; \mathrm{gl}=12$, alfa $=5 \%$

Por lo tanto, no se rechaza la hipótesis nula y se dice que existe suficiente evidencia estadística para concluir que no hay una asociación entre la antigüedad del mercado y el número de empleados

Cuadro 5. Número de profesionistas

\begin{tabular}{|c|c|c|c|c|c|c|c|c|}
\hline & $\begin{array}{l}\text { Cuantos } \\
\text { profesionistas } \\
\text { tiene la } \\
\text { empresa }\end{array}$ & $\begin{array}{l}\text { Numero de } \\
\text { profesionistas } \\
\text { en corte }\end{array}$ & $\begin{array}{l}\text { Numero de } \\
\text { profesionistas } \\
\text { en ensamble }\end{array}$ & $\begin{array}{l}\text { Numero de } \\
\text { profesionistas } \\
\text { en lijado y } \\
\text { pintura }\end{array}$ & $\begin{array}{l}\text { Numero de } \\
\text { profesionistas } \\
\text { en empaque y } \\
\text { embalaje }\end{array}$ & $\begin{array}{l}\text { Numero de } \\
\text { profesionistas } \\
\text { en } \\
\text { administrativo }\end{array}$ & $\begin{array}{l}\text { Numero de } \\
\text { profesionistas } \\
\text { en calidad }\end{array}$ & $\begin{array}{l}\text { Numero de } \\
\text { profesionistas en } \\
\text { ventas/embarques }\end{array}$ \\
\hline Media & 1.53 & .07 & .00 & .00 & .00 & .53 & .13 & .27 \\
\hline Rango & 8 & 1 & 0 & 0 & 0 & 1 & 1 & 1 \\
\hline Suma & 23 & 1 & 0 & 0 & 0 & 8 & 2 & 4 \\
\hline
\end{tabular}

Fuente: Elaboración propia.

Como se observa en el cuadro 5, de las empresas participantes en el estudio el mayor número de profesionistas laboran en las áreas administrativas, de calidad y de ventas. El total de profesionistas en la muestra fue de $8.24 \%$ del número total de trabajadores. Esto representa una gran debilidad para las empresas, por lo que deben fortalecer las áreas de capacitación en las distintas profesiones y contratar profesionales en áreas claves como en calidad y producción más que en administración ventas. 
Cuadro 6. Producción anual.

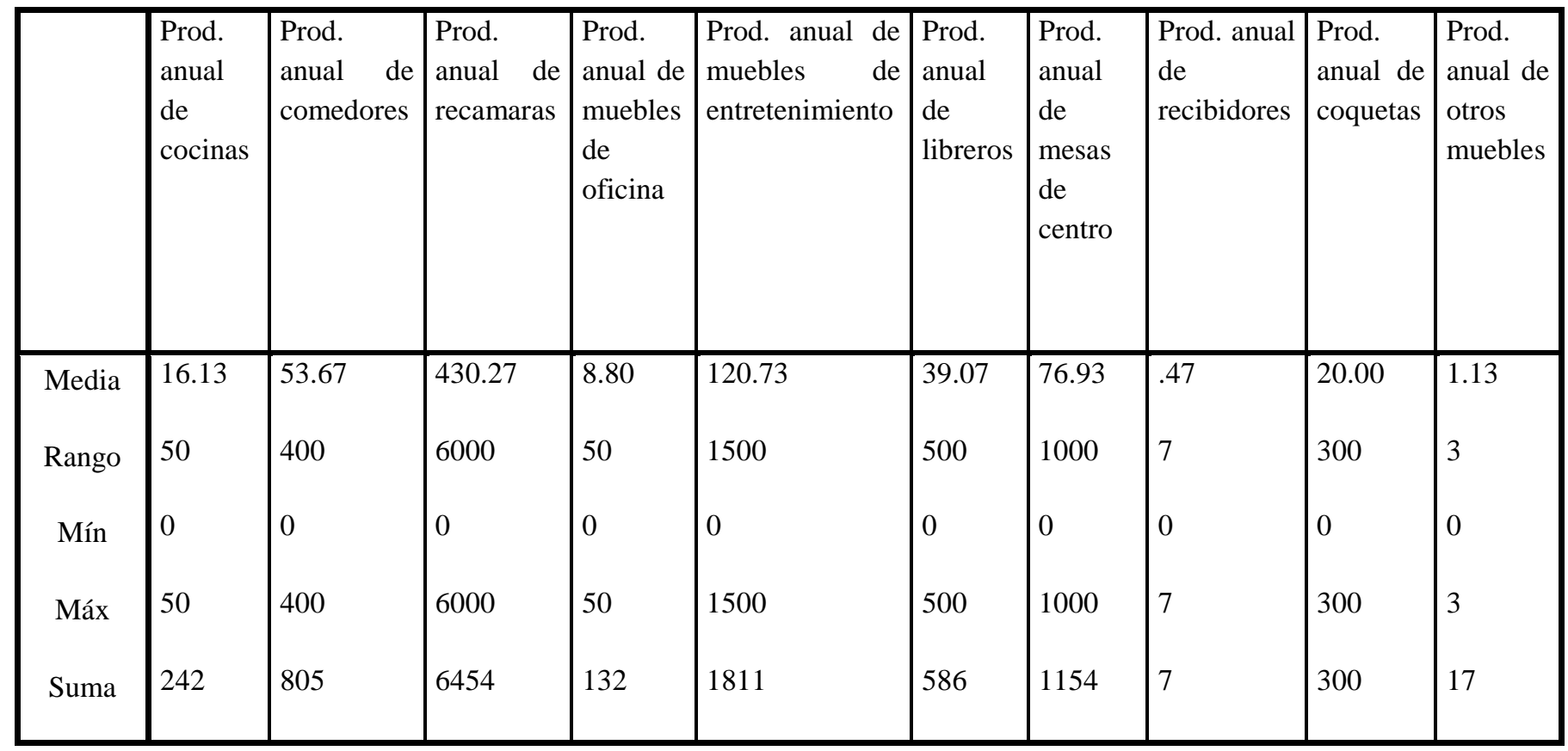

Fuente: Elaboración propia.

De las empresas que fueron muestra investigación manifestaron que la mayor producción anual es la elaboración de recamaras con una producción máxima de 6000 de por año seguido por 1500 muebles de entretenimiento anuales. Este estudio nos indica que el porcentaje menor en la producción es representado por los muebles de oficina con 132 unidades por año y recibidores con 7 unidades anuales. Como se observa en el cuadro 6.

\section{CONCLUSIÓN}

De la base de datos obtenida de las empresas que fueron objeto de estudio se clasifican como microempresas, pequeñas $\mathrm{y}$, medianas empresas. A pesar de que el número de profesionistas es limitado en respecto al total de los trabajadores, estos se concentraron en el área administrativa. Esto representa una gran debilidad para las empresas, por lo que deben fortalecer las áreas de capacitación y contratar profesionales en áreas claves como supervisión de calidad y diseño. El 93\% no cuentan con un plan estratégico establecido.

De igual manera, se observó que más del $60 \%$ no cumplen con la normatividad del permiso de uso de suelo industrial. El $100 \%$ de las empresas no tienen información del significado de la importancia, de aplicar capacitación en la norma ISO 035, vigente desde el año 2019.

A pesar de la antigüedad en el mercado las empresas el $87 \%$ no son parte de la asociación de fabricantes de muebles de Delicias. El estudio alcanzo los objetivos declarados al conocer las capacidades productivas de las industrias productoras de muebleras de madera del municipio de Delicias, Chihuahua, particularmente de las micro y las pequeñas empresas.

Entre sus principales fortalezas se encontró que las empresas que participaron en el estudio el $53 \%$ cuenta con una antigüedad de 31 a 40 años y el $7 \%$ con más de 40 años de antigüedad lo que nos demuestra que son dirigidas por 
personas con una amplia experiencia en el área, y cuentan con capacidad emprendedora y potencial para transformar los productos de madera de la mejor forma, haciéndonos ver que utilicen sus recursos y habilidades de una forma adecuada y óptima para alcanzar sus objetivos, tales como mantenerse en el mercado con los mejores diseños y con una alta calidad en su producto

Sin embargo, concluimos que, se tiene que trabajar más en sus debilidades, capacitación en diferentes áreas de la empresa, planeación estratégica e integración a las diferentes asociaciones empresariales.

\section{LITERATURA CITADA}

Araiza Garza Zochitl., Díaz Diaz Guadalupe y Garza Martinez Edna Isabel. (2007). Problemática de las Micros, Pequeñas y Medianas del Estado de Coahuila, México. XI Congreso Internacional de la Academia de Ciencias Administrativas A.C. (ACACIA), México. Universidad Autónoma de Coahulia en la facultad de Contaduría y Administración de la Unidad Norte en Monclova, Coah.

http://acacia.org.mx/busqueda/pdf/P30T 15.pdf

Carriedo Carlos (2017). Pymes mexicanas y su estrategia para 2017. Revista Forbes México.

https://www.forbes.com.mx/pymes-

mexicanas-y-su-estrategia-para-2017/

Daza Lesmes Javier (2010) Desafíos y recomendaciones a la planeación estratégica en universidades. Escuela de Medicina y Ciencias de la Salud, Universidad del Rosario, Colombia. https://doi.org/10.35362/rie5421683

Espinosa Gómez (2012). La industria y el comercio del mueble en México. Revista Internacional de investigación en mobiliario y objetos decorativos. Res Mobilis. Vol. 1, $\mathrm{n}^{\mathrm{o}}$. 1, 2012. Universidad Autónoma de México. https://doi.org/10.17811/rm.1.2012.73-87

George, D., \& Mallery, P. (2003). SPSS for Windows step by step: A simple guide and reference, 11.0 update. Obtenido de https://wps.ablongman.com/wps/media/o bjects/385.George 4answers pdf

González Estrada Ana Cristina, Jacinto Sánchez Beatriz Adriana, Salas Mónico Gildardo, Tapia Flores Vanessa Araceli, (2016). Análisis y Proyección Financiera de la industria de muebles en México. Instituto Politécnico Nacional

INEGI (2019). Resultados de la encuesta nacional sobre productividad $\mathrm{y}$ competitividad de las micro, pequeñas y medianas empresas (Enaproce) 2018. https://www.inegi.org.mx/contenidos/sal adeprensa/boletines/2019/especiales/EN APROCE2018.pdf

López Salazar Alejandra (2015). La planeación estratégica en la pequeña y mediana empresa: una revisión bibliográfica. Centro Universitario de Ciencias Económico Administrativas de la Universidad de Guadalajara. Guadalajara, México. https://dx.doi.org/10.18381/eq.v2i1.171 
Magaña Magaña José Eduardo, Baray Guerrero María del Rosario. (2003). El Desarrollo Futuro Sustentable De La Región Centro-Sur Del Estado De Chihuahua: Cluster Industria Mueblera. Universidad Autónoma De Chihuahua. Facultad De Ciencias Agrícolas y Forestales. Delicias, Chihuahua.

Revista Porte (2016). Es tiempo del mueble sustentable.

http://www.revistaporte.com/2016/12/19/ es-tiempo-del-mueble-sustentable/?cv=1

Consulado 29 de mayo 2019, 13:00 hrs.
Velarde López Elvira, Araiza Garza Zóchitl, Hernández Castro Nidia, Tobías Sierra Lluvia (2011) Estrategias de dirección y tecnologías de información en pequeñas y medianas empresas de Coahuila. Revista Internacional Administración \& Finanzas. Volumen 4. Número 1. Universidad Autónoma de Coahuila, México.

Copyright (c) 2020 Magaña Magaña José E duardo, Talamantes G onzález Thalia Fernanda, Villarreal Ramirez Victor Hugo, Kiessling Davison Christian Mauricio, Palacios Monarrez Abdoón

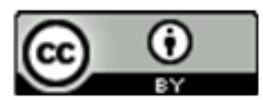

Este texto está protegido por una licencia licencia CreativeCommons 4.0.

Usted es libre para Compartir —copiar y redistribuir el $\mathrm{m}$ aterial en cualquier medio o formato-y Adaptar el documento —remezclar, transformar y crear a partir del material- para cualquier propósito, incluso para fines comerciales, siempre que cumpla la condición de:

Atribución: Usted debe dar crédito a la obra original de manera adecuada, proporcionar un enlace a la licencia, e indicar si se han realizado cambios. Puede hacerlo en cualquier forma razonable, pero no de forma tal que sugiera que tiene el apoyo del licenciante o 10 recibe por el uso que hace de la obra.

Resumendelicencia - Textocompletodelalicencia 